\title{
Construction of an Electron Bridge in Polyoxometalates/Graphene Oxide Ultrathin Nanosheets to Boost the Lithium Storage Performance
}

Jia-Nan Chang, ${ }^{\dagger a}$ Mi Zhang, ${ }^{\dagger b}$ Guang-Kuo Gao, ${ }^{\dagger a, c}$ Meng Lu,,${ }^{a}$ Yi-Rong Wang, ${ }^{a}$ Cheng Jiang, ${ }^{a}$ Shun-Li Li, ${ }^{a}$ Yifa Chen, ${ }^{* a}$ and Ya-Qian Lan ${ }^{* a, b}$

aJiangsu Key Laboratory of Biofunctional Materials, School of Chemistry and Materials Science, Nanjing Normal University, Nanjing 210023, P. R. China ${ }^{\mathrm{b} S c h o o l}$ of Chemistry, South China Normal University, Guangzhou, 510006, P. R. China

'School of Materials Science and Engineering, College of Chemical and Environmental Engineering, Harbin University of Science and Technology, Harbin, 150040, China

Jia-Nan Chang, Mi Zhang and Guang-Kuo Gao contributed equally to this work.

Correspondence and requests for materials should be addressed to Y. C (email: chyf927821@163.com) and Y.-Q. L. (email: yqlan@njnu.edu.cn) 


\section{Outline}

Figure S1 | The structural diagram of $\mathrm{MnMo}_{6}-2 \mathrm{NH}_{2}$.

Figure S2 | PXRD and Raman spectrogram of the simulated $\mathrm{MnMo}_{6}-2 \mathrm{NH}_{2}-\mathrm{GO}-\mathrm{x} \ldots .$.

Figure S3 | Characterization of $\mathrm{MnMo}_{6} / \mathrm{GO}$

Figure S4 | Characterization of GO

Figure S5 | Characterization of $\mathrm{MnMo}_{6}-2 \mathrm{NH}_{2}-\mathrm{GO}-1$ and $\mathrm{MnMo}_{6}-2 \mathrm{NH}_{2}-\mathrm{GO}-3 \ldots \ldots \ldots$

Figure S6 | Characterization of $\mathrm{MnMo}_{6}-2 \mathrm{NH}_{2}-\mathrm{GO}-4$ and $\mathrm{MnMo}_{6}-2 \mathrm{NH}_{2}-\mathrm{GO}-5$. ...

Figure S7 | XPS spectra of $\mathrm{MnMo}_{6}-2 \mathrm{NH}_{2}-\mathrm{GO}-2$ after discharged at $0.01 \mathrm{~V}$.

Figure S8 | $\mathrm{N}_{2}$ sorption measurements and pore volumes.

Figure S9 | Electrochemical performances

Figure S10 | Electrochemical performances

Figure S11 | Electrochemical performance.

Table S1 | Comparison of $\mathrm{MnMo}_{6}-2 \mathrm{NH}_{2}-\mathrm{GO}$ hybrid materials with different $\mathrm{MnMo}_{6}-2 \mathrm{NH}_{2}$

Table S2 | Comparison of $\mathrm{MnMo}_{6}-2 \mathrm{NH}_{2}-\mathrm{GO}$ hybrid materials with different GO.....

Table S3 | Comparison of $\mathrm{MnMo}_{6}-2 \mathrm{NH}_{2}-\mathrm{GO}-2$ with other pristine GO and POMs based anodes. 


\section{Calculation of the theoretical capacities:}

The theoretical capacities were calculated according to equation S1:

$$
Q=\frac{n F}{3.6 M}=\frac{96500 n}{3.6 M} \quad \text { equation S1 }
$$

where $Q$ is the reversible charging-discharging capacity, $n$ is the number of electrons passed during the redox reaction, and $M$ is the molecular weight.

\section{Calculation of the theoretical capacities of POMs:}

When $\mathrm{Li}^{+}$intercalate/ deintercalate into the structure of $\mathrm{MnMo}_{6}-2 \mathrm{NH}_{2}$, we based on the redox reactions of metal ions (Mo) which are due to the intercalation mechanism for $\mathrm{Li}$ storage (equations S2) and the XPS results in Fig. $2 \mathrm{~g}$.

$$
M \Phi^{6+} \leftrightarrow M \Phi^{4+} \quad \text { equation S2 }
$$

If $6 \mathrm{Mo}^{6+}$ are all reduced to $\mathrm{Mo}^{4+}$, maximum of $n=12, Q_{\mathrm{POM}}=485 \mathrm{mAh} \mathrm{g}^{-1}$. According to the experiment, we can calculate the content of the POMs is about $70 \%$, $\mathrm{Q}_{(\text {MnMo6-2NH2-GO-2) }}=485 \times 70 \%=339.5 \mathrm{mAh} \mathrm{g}^{-1}$. 
Materials: N-methyl-2-pyrrolidone $\left(\mathrm{C}_{5} \mathrm{H}_{9} \mathrm{NO}\right)$, Poly(vinylidene fluoride $)\left(-\left(\mathrm{CH}_{2} \mathrm{CF}_{2}\right)_{n-}\right)$, Toner $(\mathrm{C})$ and Tetrabutylammonium bromide $\left(\mathrm{C}_{16} \mathrm{H}_{36} \mathrm{BrN}\right)$ were purchased from Aladdin Reagent Co., Ltd. Sodium molybdate $\left(\mathrm{NaMoO}_{4} \cdot 2 \mathrm{H}_{2} \mathrm{O}\right)$, potassium permanganate $\left(\mathrm{KMnO}_{4}\right)$, hydrochloric acid $(\mathrm{HCl})$, concentrated sulfuric acid $\left(\mathrm{H}_{2} \mathrm{SO}_{4}\right)$, Ammonium molybdate tetrahydrate $\left(\left(\mathrm{NH}_{4}\right)_{6} \mathrm{Mo}_{7} \mathrm{O}_{24} \cdot 4 \mathrm{H}_{2} \mathrm{O}\right)$, hydrogen peroxide $\left(\mathrm{H}_{2} \mathrm{O}_{2}\right)$ and Tris(hydroxymethyl)methyl aminomethane $\left(\mathrm{C}_{4} \mathrm{H}_{11} \mathrm{NO}_{3}\right)$ were purchased from Sinopharm Chemical Reagent Co., Ltd. Reagent Co., Ltd. Manganese (III) acetate hydrate $\left(\mathrm{Mn}\left(\mathrm{CH}_{3} \mathrm{COO}\right)_{3} .2 \mathrm{H}_{2} \mathrm{O}\right)$ and Manganese sulfate $\left(\mathrm{MnSO}_{4}\right)$ were purchased from Alfa Aesar Chemical Co., Ltd. The electrolytes containing $1 \mathrm{M}$ lithium hexafluorophosphate $\left(\mathrm{LiPF}_{6}\right)$, ethyl carbonate $\left(\mathrm{C}_{3} \mathrm{H}_{4} \mathrm{O}_{3}\right)$ and dimethyl carbonate $\left(\mathrm{C}_{3} \mathrm{H}_{6} \mathrm{O}_{3}\right)$ (volume ratio of 1:1) were purchased from Duoduo Chemical Reagent Co., Ltd.

Characterization: Powder X-ray diffraction (PXRD) experiments were recorded on a Bruker D8 Advance (operating at $40 \mathrm{kV}$ and $20 \mathrm{~mA}$ ) with Ni-filtered $\mathrm{Cu}$ Ka radiation at $1.5406(\AA)$ with a speed of $5^{\circ} \mathrm{min}^{-1}$. SEM images were detected from a FEI NOVA NANO 430 Field Emission Scanning Electron Microscope equipped with an Oxford Energy Dispersive X-ray spectroscopy. TEM images and STEM-HAADF images coupled to EDS elemental mapping were collected on a JEOL JEM-2100 electron microscope at $200 \mathrm{kV}$ equipped with an Oxford Energy Dispersive X-ray spectroscopy. $\mathrm{N}_{2}$ sorption measurements were carried out on a Micromeritics ASAP 2460 system at $77 \mathrm{~K}$. During the $\mathrm{N}_{2}$ sorption tests, the samples were first degassed at $120{ }^{\circ} \mathrm{C}$ for 8 h. X-ray photoelectron spectroscopy (XPS) was measured on the instrument model Phi 5000 versaprobe. 

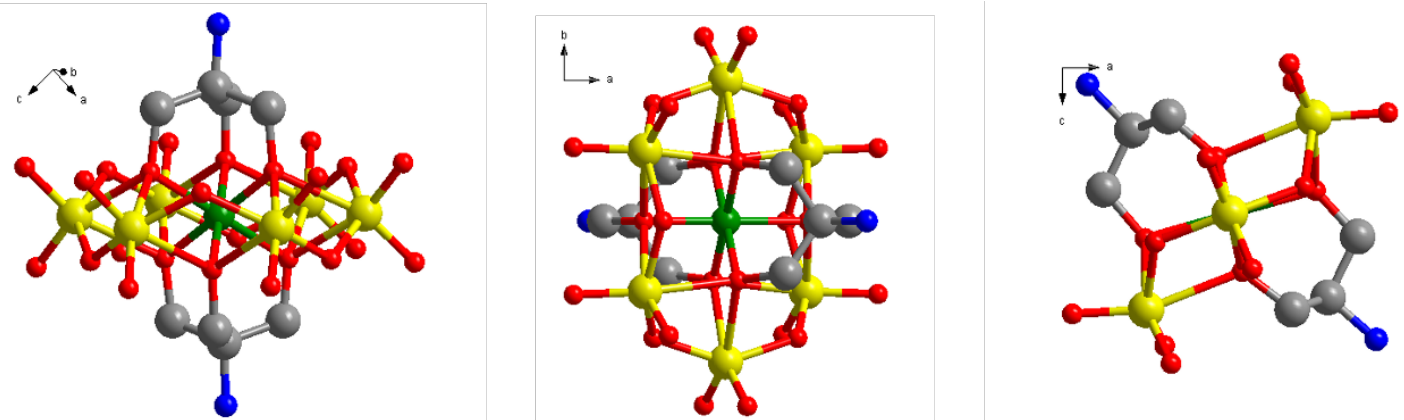

$\mathrm{Mo}$
$8 \mathrm{Mn}$
$\mathrm{O}$
$\mathrm{C}$

Figure S1. The structural diagram of $\mathrm{MnMo}_{6}-\mathrm{NH}_{2}$. 

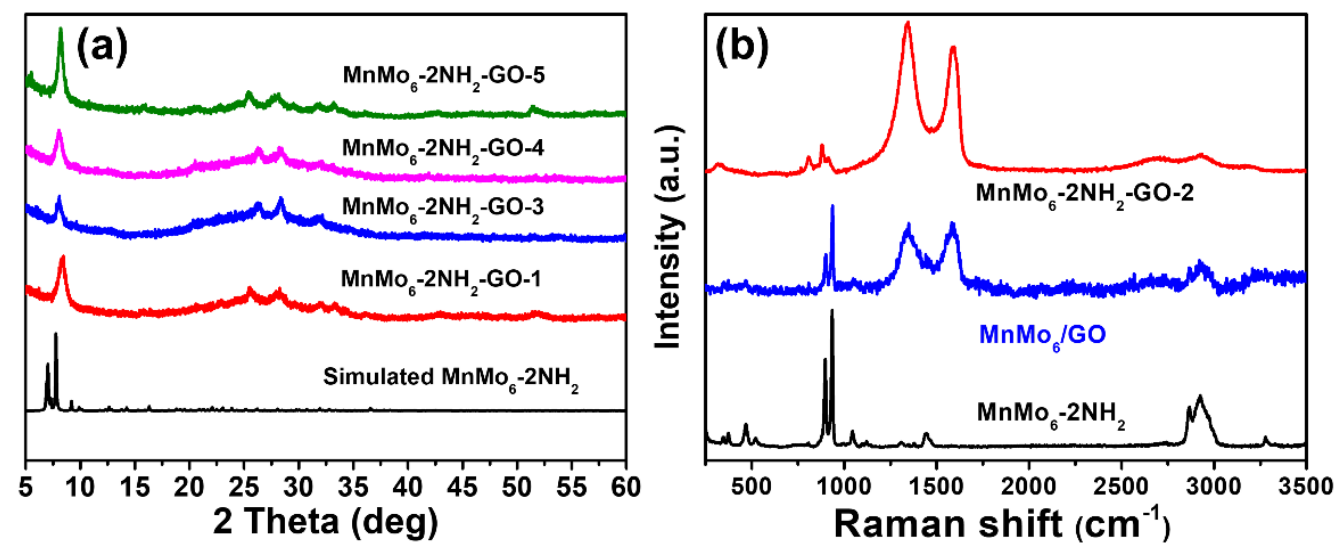

Figure S2. PXRD patterns and Raman spectra of MnMo6-2NH - GO-x (x= 1 - 5).

(a) PXRD patterns of $\mathrm{MnMo}_{6}-2 \mathrm{NH}_{2}-\mathrm{GO}-\mathrm{x}$ ( $\left.\mathrm{x}=1-5\right)$. (b) Raman spectra of MnMo $6-2 \mathrm{NH}_{2}-\mathrm{GO}-2, \mathrm{MnMo}_{6}-\mathrm{NH}_{2}$ and $\mathrm{MnMo}_{6} / \mathrm{GO}$. 

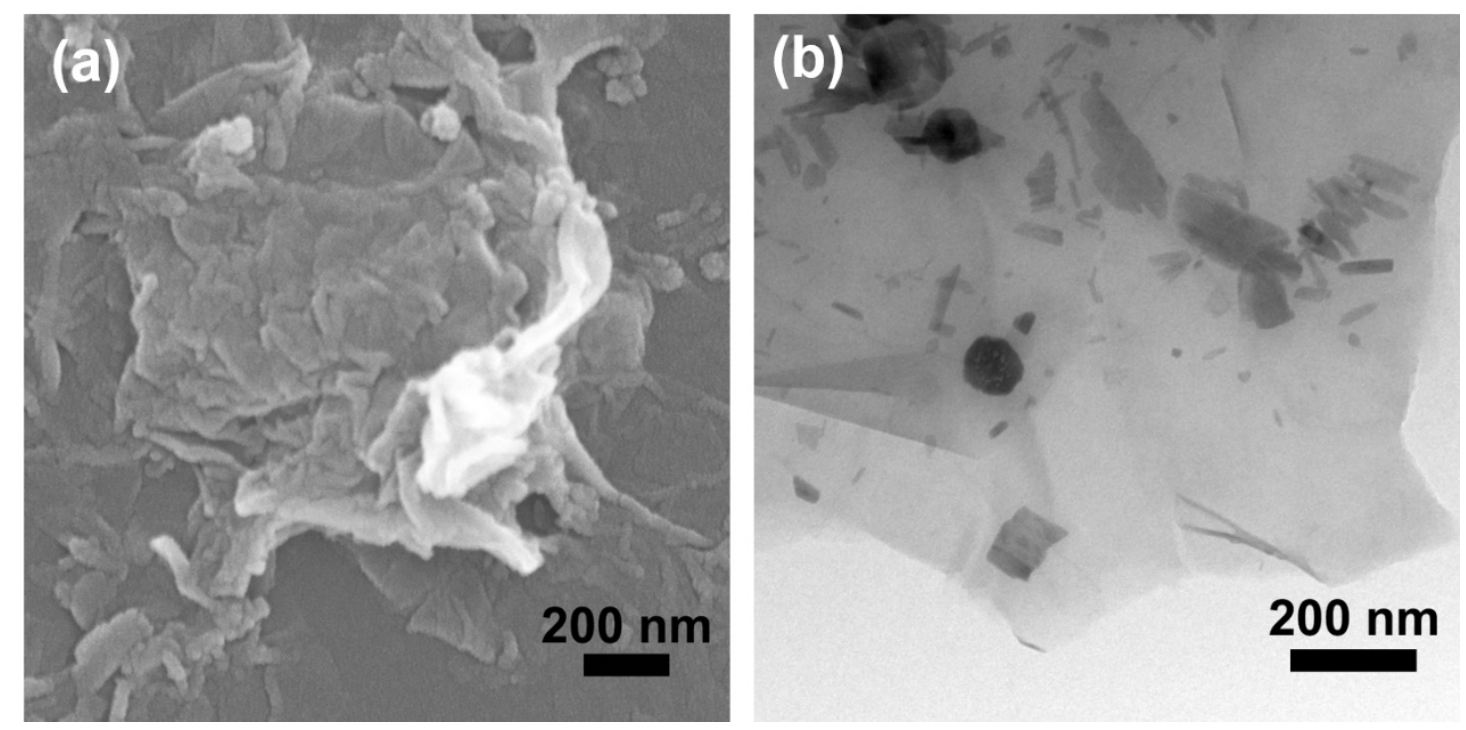

Figure S3. SEM and TEM images of MnMor/GO. (a) SEM image. (b) TEM image. 

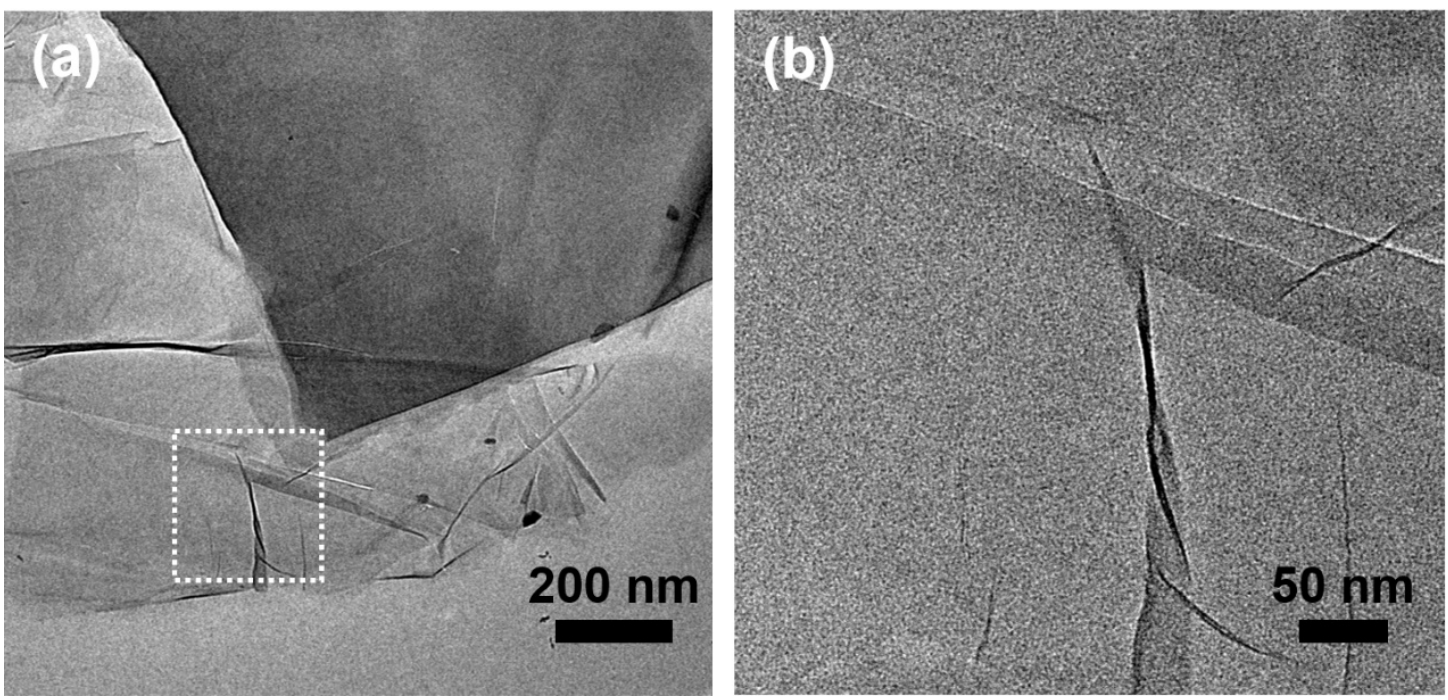

Figure S4. TEM images of GO. (a) TEM image. (b) The enlarged place in (a). 

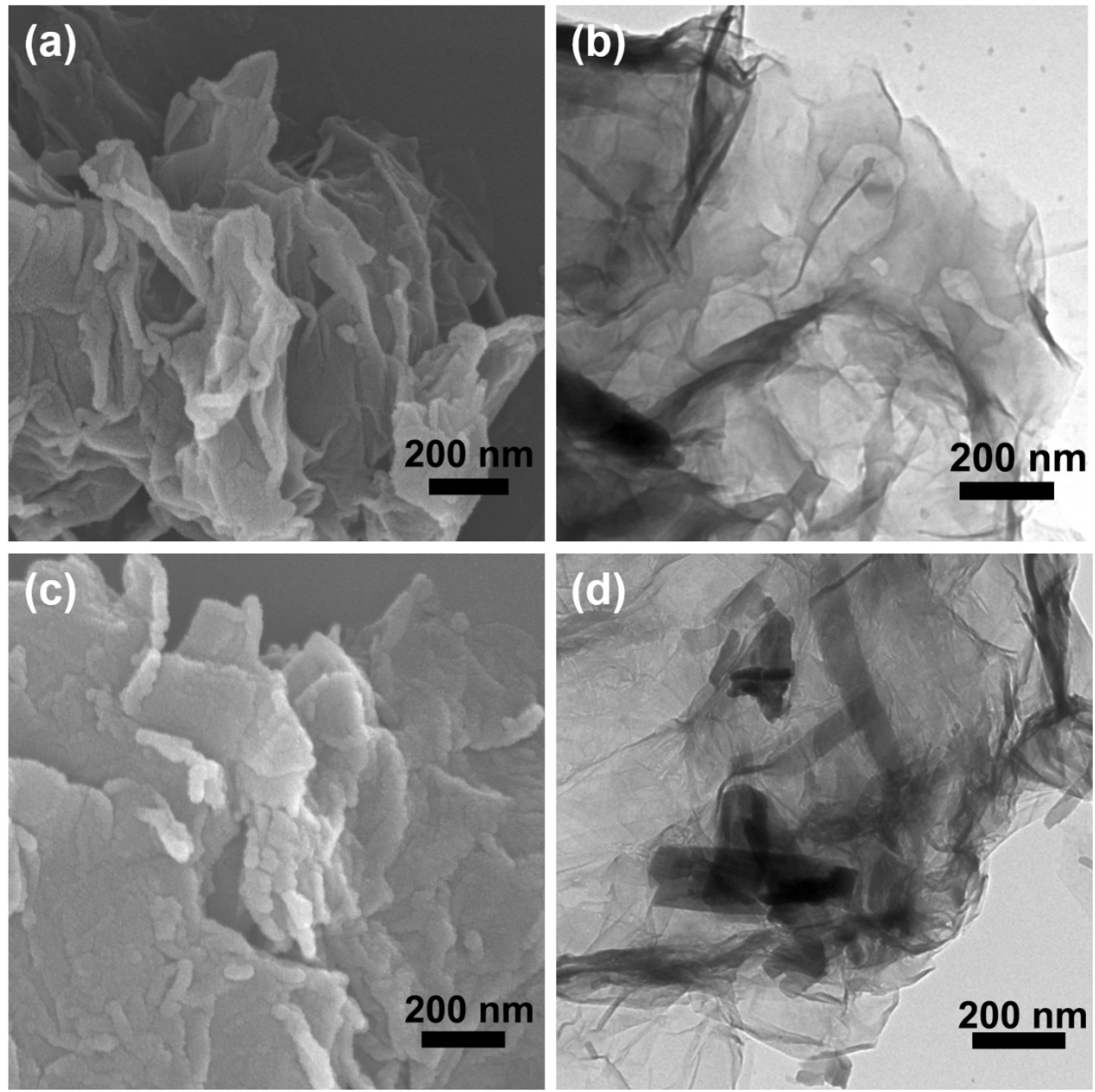

Figure S5. Morphology characterization of $\mathrm{MnMo}_{6}-\mathrm{NH}_{2}-\mathrm{GO}-1$ and MnMo6-2NH 2 -GO-3. (a, b) SEM and TEM images of MnMo6-2NH $\mathrm{N}_{2}-\mathrm{GO}-1$. (c, d) SEM and TEM images of $\mathrm{MnMo}_{6}-2 \mathrm{NH}_{2}-\mathrm{GO}-3$. 

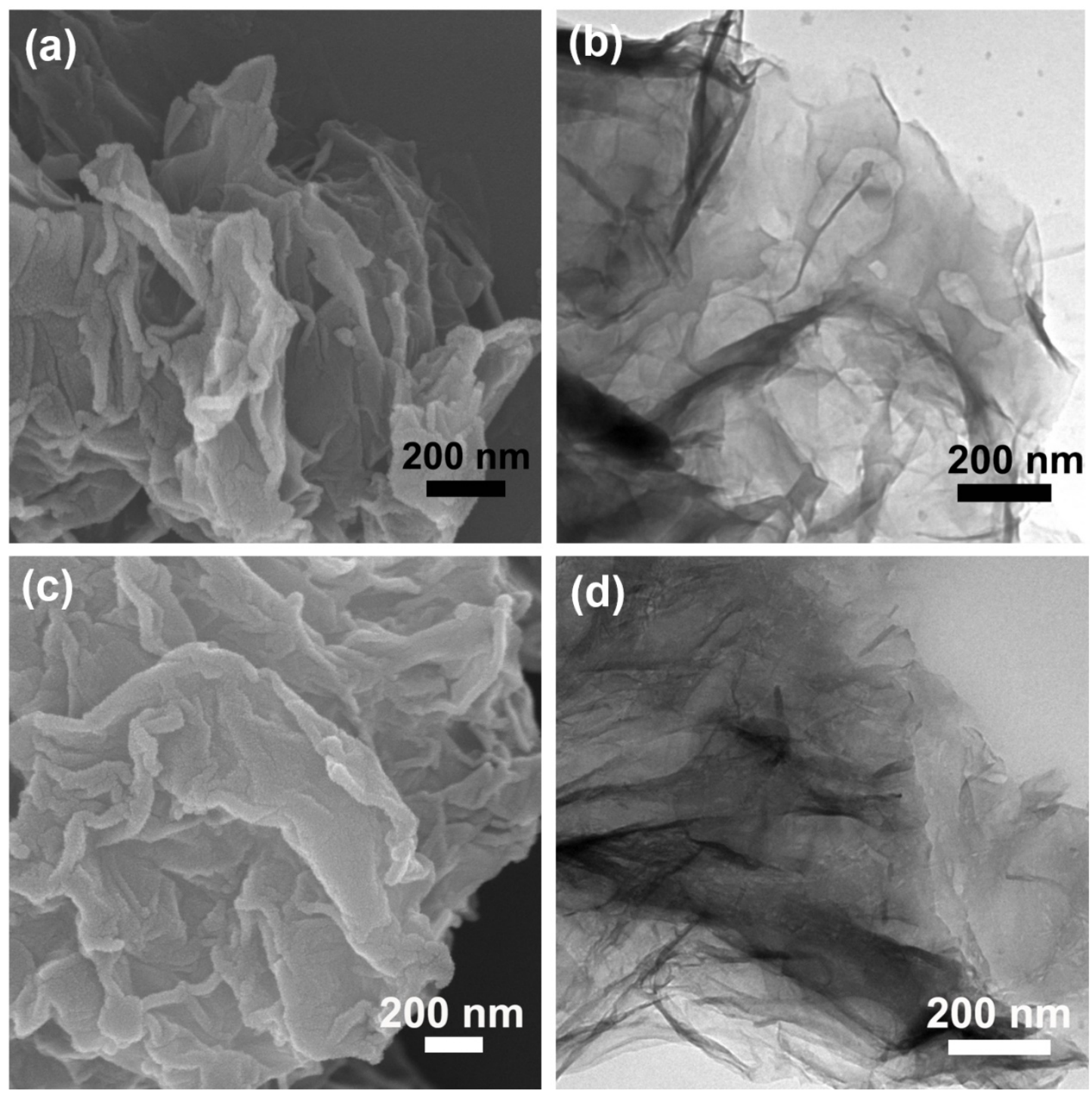

Figure S6. Morphology characterization of $\mathrm{MnMo}_{6}-\mathrm{NH}_{2}-\mathrm{GO}-4$ and MnMo6-2NH2-GO-5. (a, b) SEM and TEM images of $\mathrm{MnMo}_{6}-2 \mathrm{NH}_{2}-\mathrm{GO}-4$. (c, d) SEM and TEM images of $\mathrm{MnMo}_{6}-2 \mathrm{NH}_{2}-\mathrm{GO}-5$. 

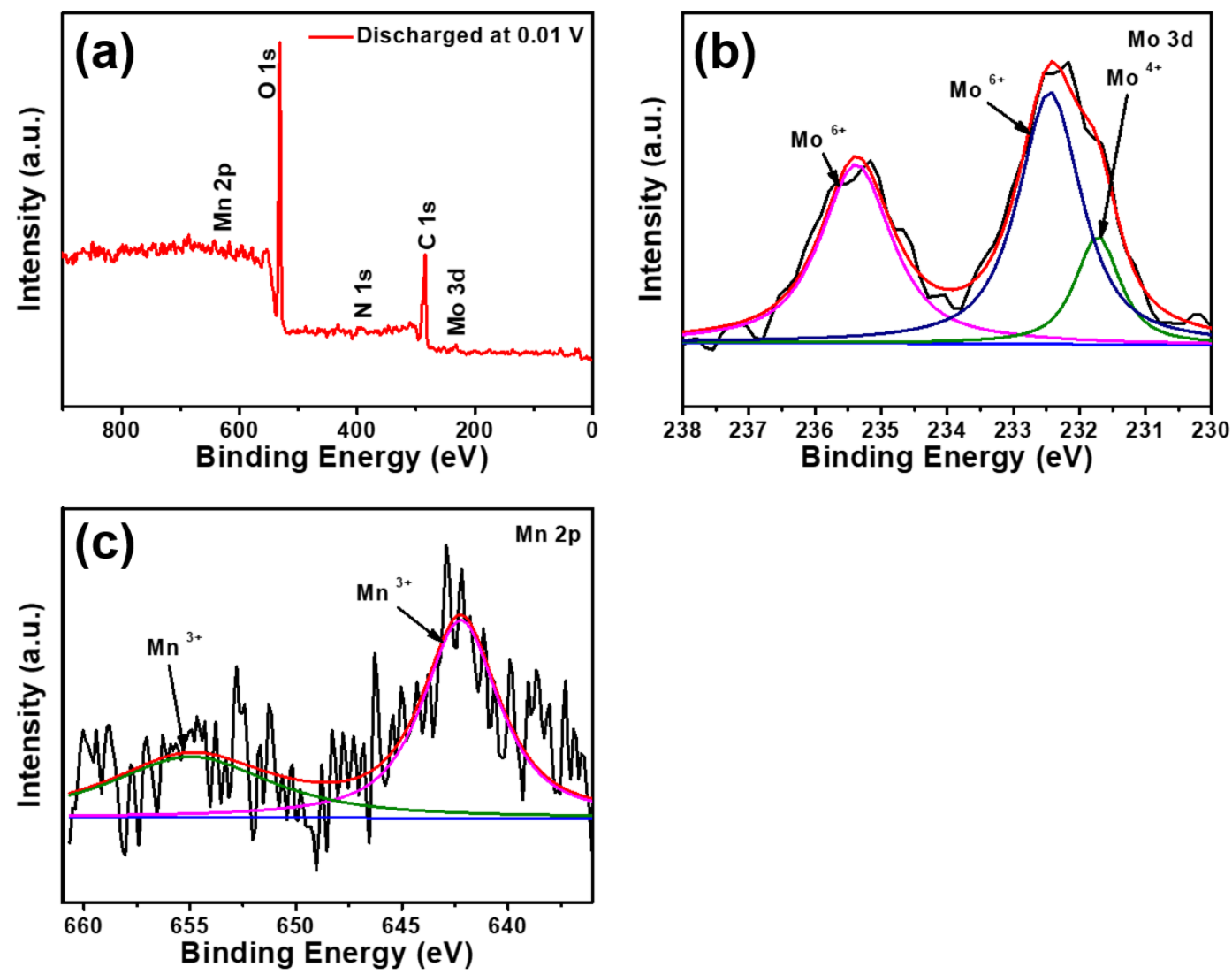

Figure S7. XPS spectra of $\mathrm{MnMo}_{6}-\mathrm{NH}_{2}-\mathrm{GO}-2$ after discharged at $0.01 \mathrm{~V}$. (a) XPS spectra. (b) Mo 3d. (c) Mn 2p. 

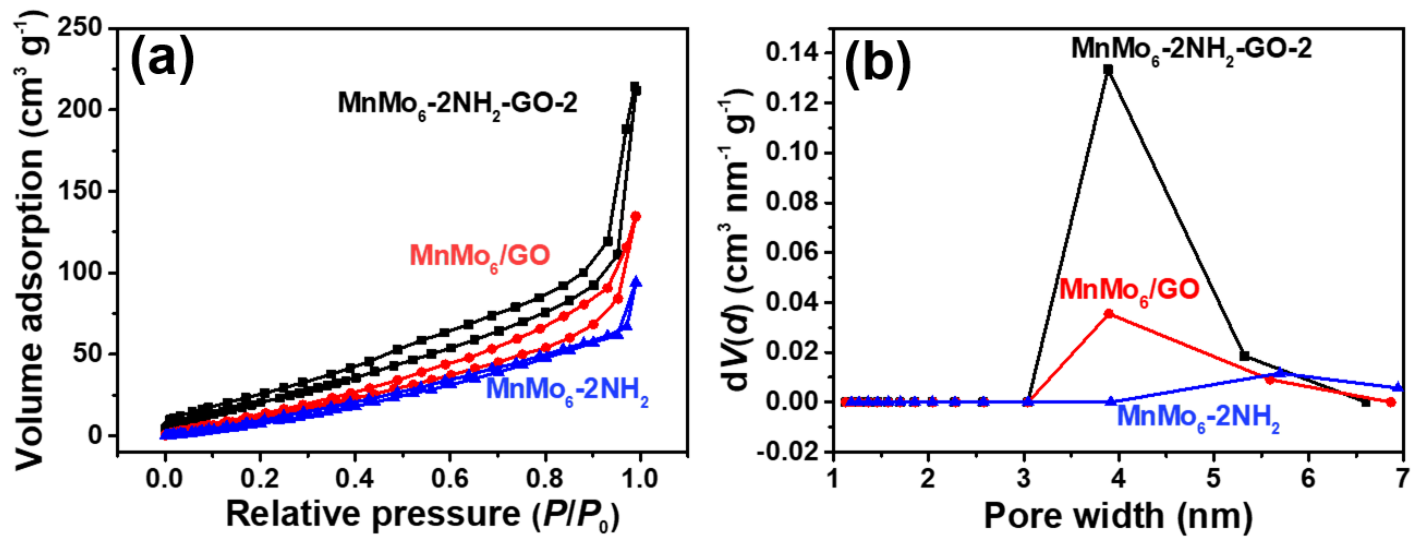

Figure S8. $\mathrm{N}_{2}$ sorption tests of $\mathrm{MnMo}_{6}-\mathrm{NH}_{2}-\mathrm{GO}-2, \mathrm{MnMo}_{6} / \mathrm{GO}$ and MnMo6-2NH2 . (a) $\mathrm{N}_{2}$ sorption curves (b) Pore size distribution curves. 

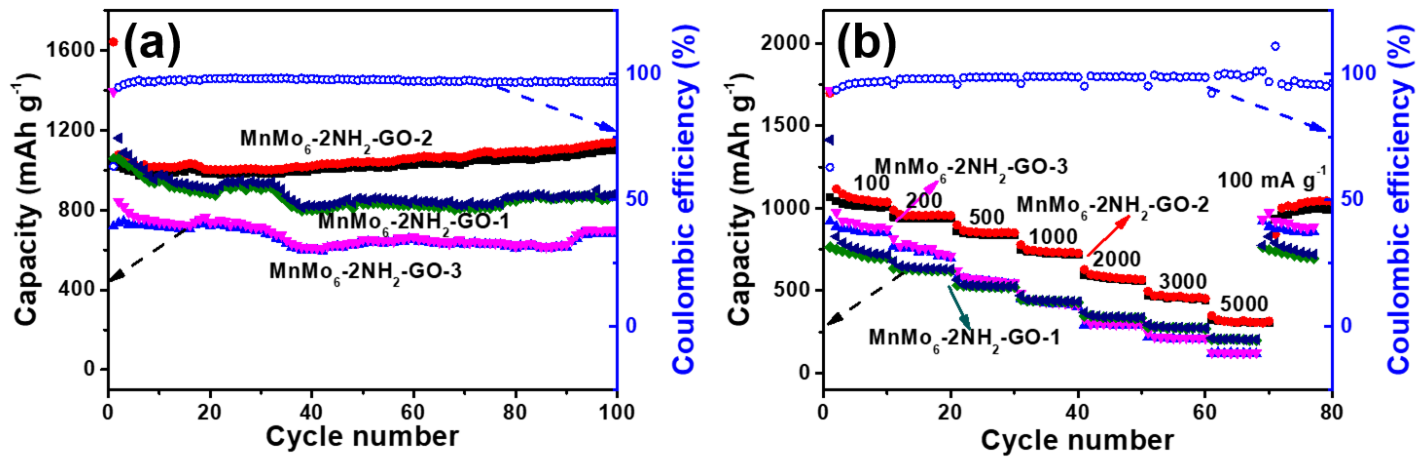

Figure S9. Electrochemical performances of $\mathrm{MnMo}_{6}-\mathrm{NH}_{2}-\mathrm{GO}-\mathrm{x}(\mathrm{x}=1,2$ and 3).

(a) Cycling performances at a current density of $100 \mathrm{~mA} \mathrm{~g}{ }^{-1}$. (b) Cycling performances at various current densities. 

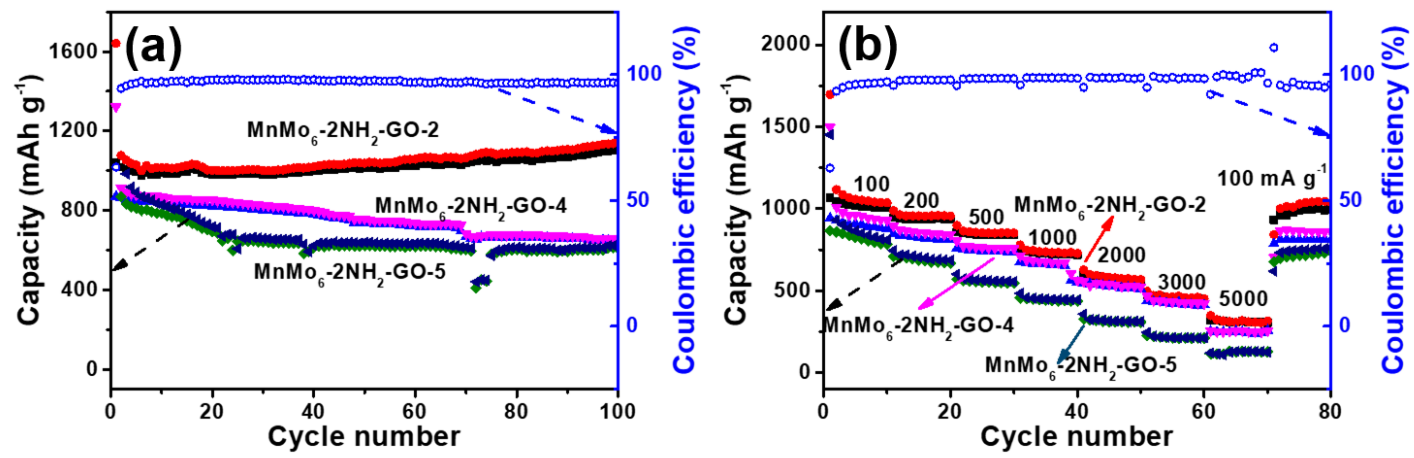

Figure S10. Electrochemical performances of $\mathrm{MnMo}_{6}-\mathrm{NH}_{2}-\mathrm{GO}-\mathrm{x}(\mathrm{x}=\mathbf{2 , 4}$ and 5)

(a) Cycling performances at a current density of $100 \mathrm{~mA} \mathrm{~g}^{-1}$. (b) Cycling performances at various current densities. 
(a)

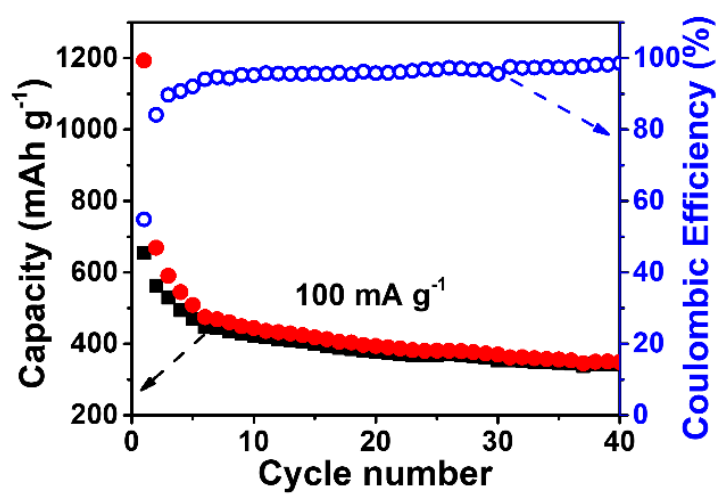

(b)

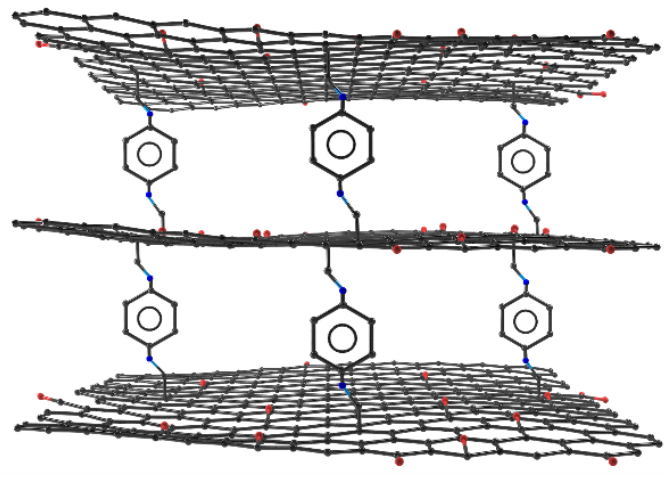

Figure S11. Electrochemical cycling performance of PDA-GO-2. (a) The cycling performances of PDA-GO-2 at a current density of $100 \mathrm{~mA} \mathrm{~g}^{-1}$. (b) The schematic representation of PDA-GO-2. 
Table S1. Cycling performances of $\mathrm{MnMo}_{6}-\mathrm{NH}_{2}-\mathrm{GO}-\mathrm{x}(\mathrm{x}=1,2$ and 3)

\begin{tabular}{lcccc}
\hline & $\begin{array}{c}\mathrm{MnMo}_{6}-\mathrm{NH}_{2} \\
\left(\mathrm{mg} \mathrm{mL}^{-1}\right)\end{array}$ & $\mathrm{GO}$ & $\mathrm{CD}$ & Cycles/RC \\
\hline $\mathrm{MnMo}_{6}-\mathrm{NH}_{2}-\mathrm{GO}-1$ & 7.55 & 1 & $\left(\mathrm{~mA} \mathrm{~mL}^{-1}\right)$ & 100 \\
\hline $\mathrm{MnMo}_{6}-\mathrm{NH}_{2}-\mathrm{GO}-2$ & 15.1 & 1 & 100 & $100 / 817$ \\
\hline $\mathrm{MnMo}_{6}-2 \mathrm{NH}_{2}-\mathrm{GO}-3$ & 30.2 & 1 & 100 & $100 / 1143$ \\
\hline
\end{tabular}

CD: Current density. RC: Reversible capacity 
Table S2. Comparison of $\mathrm{MnMo}_{6}-\mathrm{NH}_{2}-\mathrm{GO}$ hybrid materials with different GO contents.

\begin{tabular}{ccccc}
\hline Samples & $\begin{array}{c}\mathrm{MnMo}_{6}-2 \mathrm{NH}_{2} \\
\left(\mathrm{mg} \mathrm{mL}^{-1}\right)\end{array}$ & $\mathrm{GO}$ & $\mathrm{CD}$ & Cycles / RC \\
$\left(\mathrm{mg} \mathrm{mL}^{-1}\right)$ & 1 & 100 & $100 / 1143$ \\
\hline $\mathrm{MnMo}_{6}-2 \mathrm{NH}_{2}-\mathrm{GO}-2$ & 15.1 & 0.5 & 100 & $100 / 620$ \\
\hline $\mathrm{MnMo}_{6}-2 \mathrm{NH}_{2}-\mathrm{GO}-4$ & 15.1 & 2 & 100 & $100 / 610$ \\
\hline $\mathrm{MnMo}_{6}-2 \mathrm{NH}_{2}-\mathrm{GO}-5$ & 15.1 & & \\
\hline
\end{tabular}

CD: Current density. RC: Reversible capacity 
Table S3. The battery performances of various POMs based anode materials

\begin{tabular}{|c|c|c|c|c|}
\hline Materials & $\mathrm{CD}$ & $\mathrm{RC}\left(\mathrm{mAh} \mathrm{g}^{-1}\right)$ & $\operatorname{AMR}(\%)$ & Ref. \\
\hline $\mathrm{MnMo}_{6}-2 \mathrm{NH}_{2}-\mathrm{GO}-2$ & $100 \mathrm{~mA} \mathrm{~g}^{-1}$ & 1143 & 70 & This work \\
\hline $\mathrm{PMo}_{12}-\mathrm{PPy} / \mathrm{RGO}$ & $100 \mathrm{~mA} \mathrm{~g}^{-1}$ & 1082 & 70 & 1 \\
\hline Pyrene-Anderson-CNTs & $0.5 \mathrm{~mA} \mathrm{~cm}^{-2}$ & 665 & 30 & 2 \\
\hline $\mathrm{Na}_{3}\left[\mathrm{AlMo}_{6} \mathrm{O}_{24} \mathrm{H}_{6}\right] / \mathrm{KB}$ & $17 \mathrm{~mA} \mathrm{~g}^{-1}$ & 437 & 72 & 3 \\
\hline $\mathrm{Fe} / \mathrm{Co}-\mathrm{BTC}$ & $20 \mathrm{~mA} \mathrm{~g}^{-1}$ & 639 & 70 & 4 \\
\hline $\mathrm{Li}_{7}\left[\mathrm{~V}_{15} \mathrm{O}_{36}\left(\mathrm{CO}_{3}\right)\right]$ & $50 \mathrm{~mA} \mathrm{~g}^{-1}$ & 250 & 70 & 5 \\
\hline$\left[\mathrm{MnMo}_{6} \mathrm{O}_{24}\right]_{9} / \mathrm{SWNTs}$ & $0.5 \mathrm{~mA} \mathrm{~cm}^{-2}$ & 932 & 50 & 6 \\
\hline SWNTs/Py-SiW 11 & $0.5 \mathrm{~mA} \mathrm{~cm}^{-2}$ & 580 & 30 & 7 \\
\hline $\mathrm{Mo}_{6} \mathrm{O}_{18}-\mathrm{SCN}$ & $50 \mathrm{~mA} \mathrm{~g}^{-1}$ & 876 & 40 & 8 \\
\hline POMOF-1 & $1.25 \mathrm{C}$ & 350 & 65 & 9 \\
\hline
\end{tabular}


EDAG

$100 \mathrm{~mA} \mathrm{~g}^{-1}$

390

80

10

graphene foam (GF)

$4 \mathrm{~A} \mathrm{~g}^{-1}$

150

100

11

CD: Current density. RC: Reversible capacity. AMR: Active material ratio 
1. Zhang, M.; Wei, T.; Zhang, A. M.; Li, S.-L.; Shen, F.-C.; Dong, L.-Z.; Li, D.-S.; Lan, Y.-Q., Polyoxomolybdate-polypyrrole/reduced graphene oxide nanocomposite as high-capacity electrodes for lithium storage. ACS Omega 2017, 2, 5684-5690.

2. Huang, L.; Hu, J.; Ji, Y.; Streb, C.; Song, Y.-F., Pyrene-Anderson-modified CNTs as anode materials for lithium-ion batteries. Chem. -A Eur. J. 2015, 21, 18799-18804.

3. Ni, E.; Uematsu, S.; Sonoyama, N., Anderson type polyoxomolybdate as cathode material of lithium battery and its reaction mechanism. J. Power Sources 2014, 267, 673-681.

4. Xu, X.; Chen, S.; Chen, Y.; Sun, H.; Song, L.; He, W.; Wang, X., Polyoxometalate cluster-incorporated metal-organic framework hierarchical nanotubes. Small 2016, 12, 2982-2990.

5. Chen, J.-J.; Symes, M. D.; Fan, S.-C.; Zheng, M.-S.; Miras, H. N.; Dong, Q.-F.; Cronin, L., High-Performance polyoxometalate-based cathode materials for rechargeable lithium-ion batteries. Adv. Mater. 2015, 27, 4649-4654.

6. Ji, Y.; Hu, J.; Huang, L.; Chen, W.; Streb, C.; Song, Y.-F., Covalent attachment of Anderson-type polyoxometalates to single-walled carbon nanotubes gives enhanced performance electrodes for lithium ion batteries. Chem. -A Eur. J. 2015, 21, 6469-6474.

7. Ma, D.; Liang, L.; Chen, W.; Liu, H.; Song, Y.-F., Covalently tethered polyoxometalate-pyrene hybrids for noncovalent sidewall functionalization of single-walled carbon nanotubes as high-performance anode material. Adv. Funct. Mater. 2013, 23, 6100-6105.

8. Nasim Khan, R. N.; Mahmood, N.; Lv, C.; Sima, G.; Zhang, J.; Hao, J.; Hou, Y.; Wei, Y., Pristine organo-imido polyoxometalates as an anode for lithium ion batteries. RSC Adv. 2014, 4, 7374-7379.

9. Yue, Y.; Li, Y.; Bi, Z.; Veith, G. M.; Bridges, C. A.; Guo, B.; Chen, J.; Mullins, D. R.; Surwade, S. P.; Mahurin, S. M.; Liu, H.; Paranthaman, M. P.; Dai, S., A POM-organic framework anode for Li-ion battery. J. Mater. Chem. A. 2015, 3, 22989-22995.

10. Xie, J.; Zhang, Y.; Han, Y.; Li, C., High-Capacity Molecular Scale Conversion anode enabled by hybridizing cluster-type framework of high loading with amino-functionalized graphene. ACS Nano 2016, 10, 5304-5313.

11. Guo, H.; Long, D.; Zheng, Z.; Chen, X.; Ng, A. M. C.; Lu, M., Defect-enhanced performance of a 3D graphene anode in a lithium-ion battery. Nat. Nanotechnol. 2017, 28, 505402. 